\title{
ON THE ASYMPTOTIC PERFORMANCE OF MIMO CORRELATED RICEAN CHANNELS
}

\author{
Julien Dumont ${ }^{12}$, Philippe Loubaton ${ }^{2}$, Samson Lasaulce ${ }^{3}$ and Mérouane Debbah ${ }^{4}$ \\ ${ }^{1}$ France Telecom R\&D, 38-40 Rue du General Leclerc 92794 Issy-les-Moulineaux Cedex 9, France \\ ${ }^{2}$ IGM Lab. Info., UMR-CNRS 8049, Université de Marne la Vallée, 77454 Marne-la-Vallée, France \\ ${ }^{3}$ CNRS-LSS, 5, Rue Joliot Curie, 91192 Gif-sur-Yvette, France \\ ${ }^{4}$ Institut Eurecom, 2229 Route des Cretes, BP193, 06904 Sophia Antipolis Cedex, France \\ E-mail: \{dumont, loubaton\}@univ-mlv.fr,lasaulce@lss.supelec.fr, debbah@eurecom.fr
}

\begin{abstract}
In this paper we investigate the performance of block-fading Multiple Input Multiple Output (MIMO) Ricean correlated channels in the case where the number of transmit and receive antennas converge to infinity at the same rate. In this context we show that the expressions of classical performance indices such as the average mutual information or the output SINRs of MMSE receivers converge to deterministic expressions. The analysis determines the parameter of interest and gives insight of the effect of the channel distribution on the performance metrics.
\end{abstract}

\section{INTRODUCTION}

Since the seminal work of Telatar ([1]), the performance study of MIMO systems has generated considerable interest. In particular, various indices of performance (average mutual information, ergodic capacity, output SINRs -signal to noise plus interference ratios- of MMSE receivers) of block-fading Rayleigh (i.e. zero-mean) correlated channels have been studied extensively (see among others [2] to [6]). Despite the fact that the performance analysis of Ricean (non zero-mean) MIMO channels is of great practical interest, studied dedicated to this issue have been limited. The most significant works studied the mutual information and capacity of Ricean channels with independent identically distributed (i.i.d.) Rayleigh component (see e.g. [2] and [5] in the case of rank one line-of-sight (LOS) component, or [7],[8]). However these works do not discuss important issues such as the impact of the structure of the LOS or Rayleigh component on communications performance metrics. The purpose of this paper is to study the average mutual information and the output SINR of the MMSE receivers in block-fading Ricean MIMO channels with or without correlation on the Rayleigh component. We will only consider the case with correlation at the receiver. This case corresponds to important practical situations such as the downlink of a cellular system equipped with multiple antenna base and mobile stations. Moreover, it leads to very useful analytical expressions that have not been considered in the literature so far. As in [3], [6], [8] we study the performance indices mentioned above in the case where the number of transmit and receive antennas converge to the infinity at the same rate. The main advantage of this approach follows from the fact that, in this asymptotic regime, the expressions of the performance indices are simple and can be used in order to get some insights on the influence of the statistical properties of the channel. We mention that this asymptotic approach has not been studied in previous works in the context of Ricean channels (see e.g. the exhaustive monograph [5] in which non trivial Ricean channels are not considered). This paper is structured as follows. In section 2 we present the Rice MIMO channel model. In section 3 we present a useful technical result based on [9]. This result characterizes the behavior of the diagonal entries of $\left(\boldsymbol{\Sigma} \boldsymbol{\Sigma}^{H}+\sigma^{2} I\right)^{-1}$ where $\Sigma$ is a non centered random matrix of increasing dimensions with independent (but non necessarily identically distributed) entries. The result is then used to derive approximate expressions of the average mutual information and MMSE output SINRs. In section 4 we use these expressions in order to study the impact of channel statistics on mutual information and the (uncoded) bit error rate at the MMSE receiver output. In particular we identify some propagation conditions under which the considered performance indices are optimum. Finally we provide in section 5 some numerical evaluations intended to show that the asymptotic regime is reached for reasonably small numbers of antennas and to illustrate some interesting behaviors of correlated MIMO Ricean channels.

In the following, upper and lower boldface symbols will be used for matrices and column vectors, respectively. $\mathbf{A}^{H}$ stands for the conjugate transpose of matrix $\mathbf{A}, \operatorname{Tr}(\mathbf{A})$ for its trace, and the mathematical expectation operator is denoted by $\mathbb{E}($.

\section{RICE CHANNEL MODEL}

We consider a wireless MIMO link characterized by the following equation:

$$
\tilde{\mathbf{y}}=\tilde{\mathbf{H}} \mathbf{x}+\tilde{\mathbf{v}}
$$

where $\mathbf{x}$ is the vector of transmitted symbols, $\tilde{\mathbf{v}}$ is a white Gaussian noise distributed as $\mathcal{N}\left(0, \sigma^{2} \mathbf{I}\right), \tilde{\mathbf{y}}$ is the received signal and $\tilde{\mathbf{H}}$ is the channel matrix. For simplicity sake, we will assume that the number of transmit and receive antennas are equal. We denote this common value by $t$. However our results can be extended to the more general case where the channel matrix is not square. We model the channel matrix $\tilde{\mathbf{H}}$ as

$$
\tilde{\mathbf{H}}=\sqrt{\frac{K}{K+1}} \tilde{\mathbf{A}}+\sqrt{\frac{1}{K+1}} \tilde{\mathbf{W}}
$$

where $\tilde{\mathbf{A}}$ is a deterministic matrix representing a line-of-sight component normalized in such a way that $\frac{1}{t} \operatorname{Tr}\left(\tilde{\mathbf{A}} \tilde{\mathbf{A}}^{H}\right)=1$. The matrix $\tilde{\mathbf{W}}$ represents the Rayleigh part of the channel and is assumed to be distributed according to the model $\tilde{\mathbf{W}}=\mathbf{R}^{1 / 2} \tilde{\mathbf{W}}_{\text {iid }}$ where $\tilde{\mathbf{W}}_{i i d}$ is an i.i.d. centered complex Gaussian matrix with variance 
$\frac{1}{t}$ entries and $\mathbf{R}$ is a positive Hermitian matrix used to model antenna correlation at the receiver. Additionally this correlation matrix verifies $\frac{1}{t} \operatorname{Tr}(\mathbf{R})=1$. This implies that $\frac{1}{t} \mathbb{E}\left[\operatorname{Tr}\left(\tilde{\mathbf{W}} \tilde{\mathbf{W}}^{H}\right)\right]=1$. The factor $K \geq 0$ is the so-called Ricean factor. It expresses the relative strength of the direct and scattered components of the received signal.

Moreover, channel state information is assumed only at the receiver end. In this case, the covariance matrix of $x$ coincides with the identity matrix $\mathbf{I}$ and as a consequence, the average mutual information per transmit antenna $C\left(\sigma^{2}\right)$ given by $C\left(\sigma^{2}\right)=$ $\frac{1}{t} \mathbb{E}\left(\log \operatorname{det}\left(\mathbf{I}+\frac{\tilde{\mathbf{H}} \tilde{\mathbf{H}}^{H}}{\sigma^{2}}\right)\right)$. Let $\mathbf{R}=\mathbf{U D} \mathbf{U}^{H}$ be the eigenvalue/eigenvector decomposition of the correlation matrix $\mathbf{R}$ (note that $\frac{1}{t} \operatorname{Tr}(\mathbf{D})$ =1) and denote by $\mathbf{H}$ the matrix $\mathbf{H}=\mathbf{U}^{H} \tilde{\mathbf{H}}$. Then it is easily seen that $\mathbf{H}=\sqrt{\frac{K}{K+1}} \mathbf{A}+\sqrt{\frac{1}{K+1}} \mathbf{W}$ where $\mathbf{A}=\mathbf{U}^{H} \tilde{\mathbf{A}}$ and $\mathbf{W}=$ $\mathbf{D}^{\frac{1}{2}} \mathbf{W}_{\text {iid }}$. The matrix $\mathbf{W}_{i i d}$ is a Gaussian i.i.d. matrix defined by $\mathbf{W}_{i i d}=\mathbf{U}^{H} \tilde{\mathbf{W}}_{i i d}$. Using the unitary invariance of $C\left(\sigma^{2}\right)$ it is straightforward to see that $C\left(\sigma^{2}\right)=\mathbb{E}\left(\log \operatorname{det}\left(\mathbf{I}+\frac{\mathbf{H H}^{H}}{\sigma^{2}}\right)\right)$. Therefore the observation equation given by (1) is strictly equivalent to the following equation

$$
\mathbf{y}=\mathbf{H} \mathbf{x}+\mathbf{v},\left(\text { where } \mathbf{v}=\mathbf{U}^{\mathbf{H}} \tilde{\mathbf{v}}, \text { and } \mathbf{y}=\mathbf{U}^{\mathbf{H}} \tilde{\mathbf{y}}\right)
$$

in terms of mutual information. This remark is very useful because the zero-mean component of $\mathbf{H}$ has independent (but not identically distributed) entries. Note that equations (1) and (3) are also equivalent in terms of output SINR for MMSE receivers where the SINR $\beta_{k}$ is defined with respect to the $k$-th component $x_{k}$ of $\mathbf{x}$. As a consequence, in all the following, we will restrict ourselves to the observation model (3).

\section{ASYMPTOTICAL PERFORMANCE OF MIMO SYSTEMS OVER NON ZERO-MEAN CHANNELS}

\subsection{A preliminary result}

As we will see in section 3.2 both mutual information and MMSE output SINRs are strongly related to the diagonal terms of matrix $\left(\mathbf{H} \mathbf{H}^{H}+\sigma^{2} \mathbf{I}\right)^{-1}$. The convergence of the diagonal entries of the matrix $\left(\mathbf{H} \mathbf{H}^{H}+\sigma^{2} \mathbf{I}\right)^{-1}$ holds under certain conditions and is an application of a theorem due to Girko [9]. In [9], Girko studied the behavior of these terms in the case where the entries $\mathbf{H}_{i, j}$ of $\mathbf{H}$ are non centered and independent (but not identically distributed) random variables of variance $\frac{1}{t} d_{i, j}^{2}$, but not necessarily Gaussian. In our context the term $d_{i, j}^{2}$ does not depend on the index $j$, which can be used to simplify the original results of [9]. We denote by $\mathbf{Q}\left(\sigma^{2}\right)$ and $\mathbf{P}\left(\sigma^{2}\right)$ the matrices defined by $\mathbf{Q}\left(\sigma^{2}\right)=$ $\left(\mathbf{H H}^{H}+\sigma^{2} \mathbf{I}\right)^{-1}$ and $\mathbf{P}\left(\sigma^{2}\right)=\left(\mathbf{I}+\frac{\mathbf{H}_{\mathbf{H}}^{\mathbf{H}}}{\sigma^{2}}\right)^{-1}$, and denote their entries by $\left(q_{i, j}\left(\sigma^{2}\right)\right)_{(i, j)=1, \ldots, t}$ and $\left(p_{i, j}\left(\sigma^{2}\right)\right)_{(i, j)=1, \ldots, t}$ respectively. We assume that $\sup _{t}\|\mathbf{A}\|<+\infty$ where $\|\mathbf{A}\|$ represents the largest singular value of $\mathbf{A}$ (the consequences of this condition are discussed in the next section). Then, under additional purely technical assumptions, it is possible to show that the normalized trace and diagonal entries of $\mathbf{Q}\left(\sigma^{2}\right)$ and $\mathbf{P}\left(\sigma^{2}\right)$ have the same behavior as deterministic quantities given in the following theorem.

Theorem 1 It exists a unique pair $\left(m\left(\sigma^{2}\right), \delta\left(\sigma^{2}\right)\right), m\left(\sigma^{2}\right)>0$, $\delta\left(\sigma^{2}\right)>0$, satisfying the following system of equations

$$
m=F_{1}(m, \delta), \delta=F_{2}(m, \delta)
$$

where the functions $F_{1}$ and $F_{2}$ are given by

$$
\begin{gathered}
F_{1}(m, \delta)=\frac{1}{t} \operatorname{Tr}\left[\left(\sigma^{2}\left(\mathbf{I}+\frac{m \mathbf{D}}{K+1}\right)+\frac{K}{K+1} \frac{\mathbf{A} \mathbf{A}^{H}}{1+\frac{\delta}{K+1}}\right)^{-1}\right] \\
F_{2}(m, \delta)=\frac{1}{t} \operatorname{Tr}\left[\mathbf{D}\left(\sigma^{2}\left(\mathbf{I}+\frac{m \mathbf{D}}{K+1}\right)+\frac{K}{K+1} \frac{\mathbf{A A}^{H}}{1+\frac{\delta}{K+1}}\right)^{-1}\right] .
\end{gathered}
$$

Denote by $\Phi\left(\sigma^{2}\right)$ and $\Psi\left(\sigma^{2}\right)$ the diagonal matrices defined by

$$
\begin{aligned}
& \boldsymbol{\Psi}\left(\sigma^{2}\right)=\left(1+\frac{\delta\left(\sigma^{2}\right)}{K+1}\right) \mathbf{I} \\
& \boldsymbol{\Phi}\left(\sigma^{2}\right)=\sigma^{2}\left(\mathbf{I}+m\left(\sigma^{2}\right) \frac{\mathbf{D}}{K+1}\right)
\end{aligned}
$$

Then, for each $k$,

$$
\begin{aligned}
& \lim _{t \rightarrow+\infty} p_{k, k}\left(\sigma^{2}\right)-\left(\boldsymbol{\Psi}\left(\sigma^{2}\right)+\frac{K}{K+1} \mathbf{A}^{H} \boldsymbol{\Phi}\left(\sigma^{2}\right)^{-1} \mathbf{A}\right)_{k, k}^{-1}=0 \\
& \lim _{t \rightarrow+\infty} q_{k, k}\left(\sigma^{2}\right)-\left(\boldsymbol{\Phi}\left(\sigma^{2}\right)+\frac{K}{K+1} \mathbf{A} \boldsymbol{\Psi}\left(\sigma^{2}\right)^{-1} \mathbf{A}^{H}\right)_{k, k}^{-1}=0
\end{aligned}
$$

and

$$
\lim _{t \rightarrow+\infty} \frac{1}{t} \operatorname{Tr}\left(\mathbf{Q}\left(\sigma^{2}\right)\right)-m\left(\sigma^{2}\right)=0
$$

\subsection{Applying Theorem 1 to the performance analysis of MIMO systems}

In this section we explain more precisely why Theorem 1 is useful to study the behavior of the average mutual information $C\left(\sigma^{2}\right)$ and the SINR's $\left(\beta_{k}\right)_{k=1, \ldots, t}$. We also establish the connections between our figures of merit and the matrices $\mathbf{Q}$ and $\mathbf{P}$ introduced in 3.1 .

\subsubsection{Mutual Information Expression}

It is well known that the derivative $C^{\prime}\left(\sigma^{2}\right)$ of $C\left(\sigma^{2}\right)$ with respect to the parameter $\sigma^{2}$ expresses as $C^{\prime}\left(\sigma^{2}\right)=-\mathbb{E}\left(\frac{1}{\sigma^{2}}-\frac{1}{t} \operatorname{Tr}\left(\mathbf{Q}\left(\sigma^{2}\right)\right)\right.$. Using (11) shows that $C^{\prime}\left(\sigma^{2}\right)$ has the same asymptotic behavior as $\frac{1}{\sigma^{2}}-m\left(\sigma^{2}\right)$. Therefore

$\lim _{t \rightarrow+\infty} C\left(\sigma^{2}\right)-\bar{C}\left(\sigma^{2}\right)=0$ where $\bar{C}\left(\sigma^{2}\right)=\int_{\sigma^{2}}^{+\infty}\left(\frac{1}{\omega^{2}}-m\left(\omega^{2}\right)\right) d \omega^{2}$

\subsubsection{SINR Expression}

In order to express the SINR $\beta_{k}$, we denote by $\mathbf{h}_{k}$ the $k$-th column of $\mathbf{H}$ and by $\mathbf{H}_{k}$ the matrix obtained from $\mathbf{H}$ by removing the column $\mathbf{h}_{k}$. It is well known that $\beta_{k}=\mathbf{h}_{k}^{H}\left(\mathbf{H}_{k} \mathbf{H}_{k}^{H}+\right.$ $\left.\sigma^{2} \mathbf{I}\right)^{-1} \mathbf{h}_{k}$. Using straightforward algebra $\beta_{k}$ can also be written as $\beta_{k}=\frac{1}{p_{k, k}\left(\sigma^{2}\right)}-1$. The multiuser interference at the output of the MMSE receiver is usually modelled by a Gaussian distributed process. As a consequence, the (uncoded) bit error rate at the output of the $t$ MMSE receivers (one for each component of $\mathbf{x}$ ) can be derived and is equal to $P_{e}=\frac{1}{t} \sum_{k=1}^{t} Q\left(\sqrt{\beta_{k}}\right)$ in the case of a $4-Q A M$ modulation for example $(Q$ is the classical Gaussian error function). Theorem 1 thus implies that

$$
\lim _{t \rightarrow+\infty}\left(P_{e}-\bar{P}_{e}\right)=0 \text { where } \bar{P}_{e}=\frac{1}{t} \sum_{k=1}^{t} Q\left(\sqrt{\gamma_{k}}\right)
$$


and where $\gamma_{k}$ is defined by $\gamma_{k}=\frac{1}{\left(\Psi\left(\sigma^{2}\right) \mathbf{I}+\mathbf{A}^{H} \boldsymbol{\Phi}\left(\sigma^{2}\right)^{-1} \mathbf{A}\right)_{k, k}^{-1}}-1$.

Remark. As the previous results derive from Theorem 1, it is important to discuss on the assumption $\sup _{t}\|\mathbf{A}\|<+\infty$. This in conjunction with the normalization constraint $\operatorname{Tr}\left(\mathbf{A} \mathbf{A}^{H}\right)=t$ implies that the rank of $\mathbf{A}$, and therefore the rank of $\tilde{\mathbf{A}}$, should increase to $+\infty$ at the same rate than the number of antennas $t$.

\section{DISCUSSION}

In this section, we analyze the results derived in the previous section. In particular, in some special cases of interest, we determine the channel parameters optimizing $\bar{C}\left(\sigma^{2}\right)$ and $\bar{P}_{e}$.

\subsection{Low SNR regime}

In this section we study the performance in the low SNR regime i.e when $\sigma^{2} \rightarrow+\infty$ (the high SNR analysis is more complicated to conduct and will be done in a future paper). For this purpose, we have to study the behavior of $\delta\left(\sigma^{2}\right)$ and $m\left(\sigma^{2}\right)$ (see equation (4)). It is easily seen that both functions are analytic at $+\infty$ and both $m \sigma^{2}$ and $\delta \sigma^{2}$ tends to 1 when $\sigma^{2} \rightarrow+\infty$.

\subsubsection{Mutual Information}

In order to characterize the behavior of $\bar{C}\left(\sigma^{2}\right)$ we need to evaluate the first three terms of the series expansion of $m$ w.r.t. $\frac{1}{\sigma^{2}}$, and use relation (12). Using the equations (4) and the normalization contraints $\frac{1}{t} \operatorname{Tr}\left(\mathbf{A} \mathbf{A}^{H}\right)=\frac{1}{t} \operatorname{Tr}(\mathbf{D})=1$ we get after some algebra a simple expression of the average mutual information:

$$
\bar{C}\left(\sigma^{2}\right)=\frac{1}{\sigma^{2}}-\frac{1}{2 \sigma^{4}}\left(\frac{2 K+1}{(K+1)^{2}}+\frac{1}{t} \operatorname{Tr}\left(\frac{K}{K+1} \mathbf{A} \mathbf{A}^{H}+\frac{1}{K+1} \mathbf{D}\right)^{2}\right)
$$

up to the $o\left(\sigma^{-4}\right)$ terms. The dominant term is of course equal to $\frac{1}{\sigma^{2}}$ and does not depend on the channel statistics. It is however interesting to discuss the behavior of the second term and to evaluate the channel parameters for which it is maximized. We first consider the case where the Ricean factor $K$ is fixed. In order to maximize the righthandside of (14) one has to minimize $\frac{1}{t} \operatorname{Tr}\left(\frac{K}{K+1} \mathbf{A} \mathbf{A}^{H}+\frac{1}{K+1} \mathbf{D}\right)^{2}$. The Jensen's inequality states that for any positive matrix $\mathbf{B}, \frac{1}{t} \operatorname{Tr}\left(\mathbf{B}^{2}\right) \geq\left(\frac{1}{t} \operatorname{Tr} \mathbf{B}\right)^{2}$ with equality if and only if $\mathbf{B}=\mathbf{I}$. Using this result for $\mathbf{B}=\frac{K}{K+1} \mathbf{A} \mathbf{A}^{H}+\frac{1}{K+1} \mathbf{D}$ and noting that $\frac{1}{t} \operatorname{Tr}(\mathbf{B})=1$ shows that the approximate mutual information is maximum if and only if $\mathbf{A}$ and $\mathbf{D}$ are related through

$$
\frac{K}{K+1} \mathbf{A} \mathbf{A}^{H}+\frac{1}{K+1} \mathbf{D}=\mathbf{I} .
$$

In this case the $o\left(\frac{1}{\sigma^{4}}\right)$ term equals $-\left(1+\frac{2 K+1}{(K+1)^{2}}\right)$. Therefore, if $K$ is not fixed, $\bar{C}$ is maximized if and only if $K=+\infty$ and $\mathbf{A} \mathbf{A}^{H}=\mathbf{I}$, which is not surprising.

Finally, we mention that if $\mathbf{A}$ and $\mathbf{D}$ are fixed, it is possible to find the value of $K$ maximizing the $O\left(\sigma^{-4}\right)$ term of $\bar{C}\left(\sigma^{2}\right)$. The optimum value is either 0 or $+\infty$ or $\frac{a-b}{c-b-1}$ where $a, b$ and $c$ represent respectively the normalized trace of the matrices $\mathbf{D}^{2}$, $\mathbf{D A A}^{H}$ and $\left(\mathbf{A} \mathbf{A}^{H}\right)^{2}$. This point is illustrated in section 5 .

\subsubsection{Bit Error Rate}

Similarly, let us study the behavior of $\bar{P}_{e}$. For this purpose we still use that $m \sigma^{2} \rightarrow 1$ and $\delta \sigma^{2} \rightarrow 1$ and deduce immediately that $p_{k, k}\left(\sigma^{2}\right)=1-\frac{1}{\sigma^{2}} \frac{K\left\|\mathbf{a}_{k}\right\|^{2}+1}{K+1}+o\left(\frac{1}{\sigma^{2}}\right)$, and that $\gamma_{k}=$ $\frac{1}{\sigma^{2}} \frac{K\left\|\mathbf{a}_{k}\right\|^{2}+1}{K+1}+o\left(\frac{1}{\sigma^{2}}\right)$, where $\mathbf{a}_{k}$ represents the $k$-th column of matrix $\mathbf{A}$. We remark that the dominant term coincides with the asymptotic value of the output SINR of the matched filter. Since the multi-user interference is dominated by the thermal noise for high noise levels, the matched filter SINR is equal to $\frac{\left\|\mathbf{h}_{k}\right\|^{2}}{\sigma^{2}}+$ $o\left(\frac{1}{\sigma^{2}}\right)$. But it is easily seen that if $t \rightarrow+\infty$ then $\left\|\mathbf{h}_{k}\right\|^{\sigma^{2}}$ converges to $\frac{K\left\|\mathbf{a}_{k}\right\|^{2}+1}{K+1}$.

We now evaluate the channel parameters for which the dominant term of $\bar{P}_{e}$ is minimum. We first note that $\frac{1}{t} \sum_{k=1}^{t} \gamma_{k}=$ $\frac{1}{\sigma^{2}}+o\left(\frac{1}{\sigma^{2}}\right)$ since $\frac{1}{t} \sum_{k}\left\|\mathbf{a}_{k}\right\|^{2}=1$. Thus the empirical mean of the SINRs does not depend on the channel parameters. As the function $t \rightarrow Q(\sqrt{t})$ is convex, $\bar{P}_{e} \geq Q\left(\sqrt{\frac{1}{t} \sum_{k}^{t} \gamma_{k}}\right)=$ $Q\left(\frac{1}{\sqrt{\sigma^{2}}}\right)$ with equality if and only if the different $\left(\gamma_{k}\right)_{k=1, \ldots, t}$ coincide that is when $\left\|\mathbf{a}_{k}\right\|=1$ for each $k$. In the low SNR regime the best MMSE performance is thus achieved if and only if the columns of $\mathbf{A}$ have all a unit norm whatever the matrix $\mathbf{D}$ is.

\subsection{Influence of $A$ in the uncorrelated case}

In this section, $\sigma^{2}$ is assumed to be fixed and the receive antennas are assumed to be uncorrelated ( $\mathbf{D}$ is equal to $\mathbf{I}$ ). In this context we want to optimize $\bar{C}$ and $\bar{P}_{e}$ w.r.t the matrix $\mathbf{A}$. The Ricean factor is also assumed to be fixed. Otherwise the best receiver performance is achieved for $K=\infty$ and $\mathbf{A}=\mathbf{I}$. As $\sigma^{2}$ is fixed in this section the corresponding notations will be temporarily removed when referring to $\bar{C}\left(\sigma^{2}\right), \delta\left(\sigma^{2}\right)$ and $m\left(\sigma^{2}\right)$. We also mention that if $\mathbf{D}=\mathbf{I}$, then functions $F_{1}$ and $F_{2}$ defined by (5), (6) coincide, and are denoted $F$ in this section. Therefore the parameters $m$ and $\delta$ defined as the unique solutions of equations (4) also coincide with the unique solution of the equation $m=F(m, m)$. In the following, $\mathbf{A}$ is a fixed LOS term and we compare the corresponding performance indices with $\bar{C}_{I}$ and $\bar{P}_{e, I}$, the performance indices corresponding to a unitary LOS matrix (it is obvious that $m$, and thus $\bar{C}$ and $\bar{P}_{e}$, only depend on $\mathbf{A} \mathbf{A}^{H}$ ). The various parameters corresponding to the matrix $\mathbf{A}$ will be indexed by $\mathbf{A}$ (e.g. function $F_{A}$ parameters $m_{A} \ldots$ ) while the parameters associated with $\mathbf{A}=\mathbf{I}$ will be indexed by $I$. Using these notations allows us to state the main result of this section.

Theorem $2 \bar{C}_{I} \geq \bar{C}_{A}$ with equality if and only if $\mathbf{A} \mathbf{A}^{H}=\mathbf{I}$ and $\bar{P}_{I, e} \leq \bar{P}_{A, e}$ with equality if and only if $\mathbf{A} \mathbf{A}^{H}=\mathbf{I}$.

Proof. Due to the lack of space, we just prove the first statement. To this end we first establish that for each $m \geq 0$, then $F_{A}(m, m) \geq F_{I}(m, m)$. Let $\left(\mu_{k}\right)_{k=1, \ldots, t}$ be the eigenvalues of $\mathbf{A A}^{H}$. Then $F_{A}(m, m)$ can be written as :

$$
F_{A}(m, m)=\frac{1}{t} \sum_{k=1}^{t}\left[\sigma^{2}\left(1+\frac{m}{K+1}\right)+\frac{K}{K+1} \frac{\mu_{k}}{\left(1+\frac{m}{K+1}\right)}\right]^{-1} .
$$

As the function $\mu \rightarrow \frac{1}{a+b \mu}$ for $a>0, b>0$ is convex the Jensen's inequality gives immediately $F_{A}(m, m) \geq F_{I}(m, m)$.

In order to complete the proof of the first statement, we remark that $F_{A}(m, m) \geq F_{I}(m, m)$ for each $m$ implies that the unique solutions $m_{A}$ and $m_{I}$ of equations $m=F_{A}(m, m)$ and $m=$ $F_{I}(m, m)$ satisfy $m_{A} \geq m_{I}$. Hence, $\frac{1}{\sigma^{2}}-m_{I} \geq \frac{1}{\sigma^{2}}-m_{A}$ for each $\sigma^{2}$. Equation (12) gives $\bar{C}_{I} \geq \bar{C}_{A}$. If the equality holds, $m_{I}$ must be equal to $m_{A}$ for each $\sigma^{2}$. Therefore, it exists $m$ for which $F_{A}(m, m)=F_{I}(m, m)$, which implies that $\mathbf{A A}^{H}=\mathbf{I}$. 


\subsection{Influence of $D$ when $A$ is unitary}

In the context of the Rayleigh channel it is known ([3]) that receive antenna correlation tends to degrade the mutual information. In the context of Ricean channels this claim is not always true. In particular we have shown that if $\sigma^{2} \rightarrow+\infty$, then the mutual information is maximum if and only if (15) holds. If $\mathbf{A}$ is not unitary it is clear that the optimum value of $\mathbf{D}$ is not $\mathbf{I}$.

On the other hand, if $\mathbf{A}$ is unitary, it seems quite relevant to check if $\mathbf{D}=\mathbf{I}$ is optimum. Although several simulation results tend to confirm this behavior, we have been able to identify counterexamples and show that there exist values of $\sigma^{2}$ and $K$ for which $\bar{P}_{e}$ is not optimum for $\mathbf{D}=\mathbf{I}$.

\section{NUMERICAL RESULTS}

In this section we illustrate our results by numerical experiments. In the following experiments the matrix $\tilde{\mathbf{A}}$ is defined as a normalized version of the matrix $\left(d\left(\theta_{1}\right), \ldots, d\left(\theta_{t}\right)\right)$ where $d(\theta)$ is the classical directional vector $d(\theta)=\left(1, e^{i \pi \cos \theta}, \ldots, e^{i \pi(t-1) \cos \theta}\right)^{T}$ and the angle of arrivals $\left(\theta_{k}\right)_{k=1, \ldots, t}$ are generated according to a uniform distribution on $[0,2 \pi]$. The matrix $\mathbf{R}$ corresponds to an exponential correlation model and the correlation coefficient is denoted $\rho$.

We first compare our approximate performance indices $\bar{C}$ and $\bar{P}_{e}$ with the corresponding values of $C$ and $P_{e}$ evaluated by MonteCarlo simulations. In figure 1 we compare $\bar{C}$ with the values of $C$ evaluated by Monte-Carlo simulations for $t=4$ and for different values of $K$ and $\rho$. As shown in figure 1 our asymptotic evaluations allows us to predict quite well the empirical results for $t=4$, which is in agreement with previous works devoted to asymptotic analyses of Rayleigh channels (see e.g. [6]). As for the comparison between $\bar{P}_{e}$ and $P_{e}$ figure 2 shows that the convergence of $\bar{P}_{e}$ toward $P_{e}$ is slower. Here, $t=8$ and $t=48$, while $K=1$ and $\rho=0.5$. This is in accordance with the work [4] in which the convergence rate of the SINR is studied in the context of uncorrelated Rayleigh channels.

We finally illustrate the result concerning the optimization of $K$ in the low SNR regime (see subsection 4.1). The predicted optimum value $K_{c}$ of $K$ is $K_{c}=0.74$, and we have evaluated $C\left(\sigma^{2}\right)$ by Monte-Carlo simulations $K=0,0.2,0.74,1,+\infty$ in the range $0 \mathrm{~dB}$ to $5 \mathrm{~dB}$. It turns out that $K=K_{c}$ corresponds to the optimum mutual information.

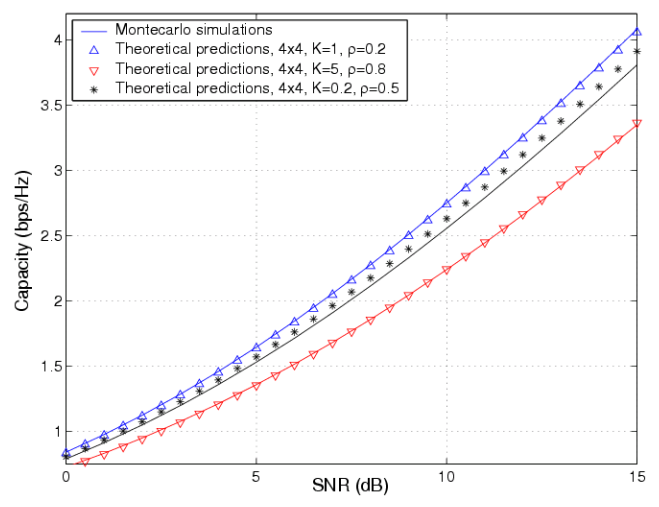

Fig. 1. Capacity : Simulation versus Theory

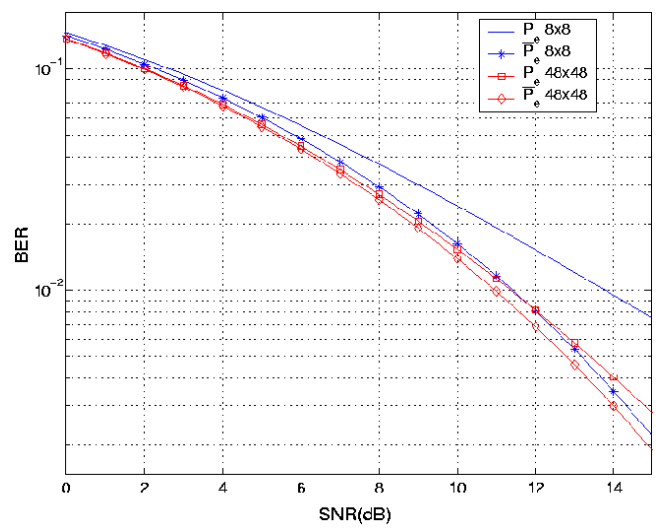

Fig. 2. Bit Error Rate : Simulation versus Theory

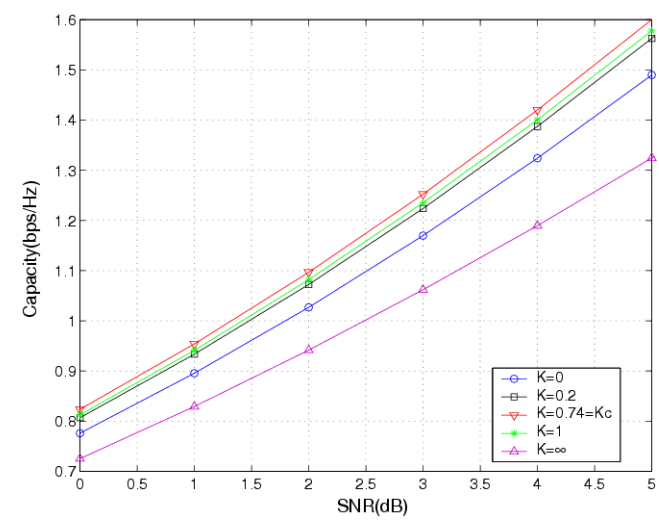

Fig. 3. Capacity for $\rho=0.5$ : Optimization for $\mathrm{K}$

\section{REFERENCES}

[1] E. Telatar, "Capacity of Multi-antenna Gaussian Channels", Europ. Trans. Telecom., vol.10, pp 585-595, Nov. 99

[2] A. Goldsmith, S.A. Jafar, N. Jindal, S. Vishwanath, "Capacity Limits of MIMO Channels", IEEE J. Sel. Areas in Comm.,vol.21, no 5,June 2003

[3] C.N. Chuah, J.M. Kahn, D.N.C. Tse, R.A. Valenzuela, "Capacity Scaling in MIMO Wireless Systems under Correlated Fading", IEEE Trans. Inf. Theo.,vol.48,no 3,pp 637-650,March 2002

[4] D.N.C. Tse, O. Zeitouni, "Linear multiuser receivers in random environments", IEEE Trans. Inf. Theo.,vol.46, no 1,pp 171-188, January 2000.

[5] A. Tulino, S. Verdu, "Random Matrix Theory and Wireless Communications", in Fondations and Trends in Communications and Information Theory, Vol.1, Now Publishers, Delft, 2004.

[6] A.L. Moustakas, A.M. Sengupta, S.H. Simon, "MIMO Capacity Through Correlated Channels in the Presence of Correlated Interference and Noise : A (Not so) Large N Analysis", Trans. on Inf. Theo., vol.49,no 10,pp 2545-2561, Oct. 03

[7] Y.H. Kim, A. Lapidoth, "On the Log-Det. of non-central Wishart Matrices", in Proc. ISIT 03, Yokohama, June 29-July 4, pp 54

[8] L. Cottatellucci, M. Debbah, "The Effect of Line of Sight Components on the Asymptotic Capacity of MIMO Systems", in Proc. ISIT 04, Chicago, June 27-July 22004

[9] V.L. Girko, "Theory of Stochastic Canonical Equations, Volume I", Chap. 7, Kluwer Acad. Publis., Dordrecht, The Netherlands, 2001. 